Y. Dong, Y. Han and Q. Ji

Nagoya Math. J.

Vol. 193 (2009), 155-167

\title{
BERNSTEIN TYPE THEOREMS FOR MINIMAL LAGRANGIAN GRAPHS OF QUATERNION EUCLIDEAN SPACE
}

\author{
YUXIN DONG, YINGBO HAN AND QINGCHUN JI
}

\begin{abstract}
In this paper, we prove some Bernstein type results for $n$ dimensional minimal Lagrangian graphs in quaternion Euclidean space $H^{n} \cong$ $R^{4 n}$. In particular, we also get a new Bernstein Theorem for special Lagrangian graphs in $C^{n}$.
\end{abstract}

\section{$\S 1$. Introduction}

The celebrated theorem of Bernstein says that the only entire minimal graphs in Euclidean 3-space are planes. This result has been generalized to $R^{n+1}$, for $n \leq 7$ and general dimension under various growth condition, see [1] and the reference therein for codimension one case. For higher codimension, the situation becomes more complicated. Due to the counterexample of Lawson-Osserman [7], the higher codimension Bernstein type result is not expected to be true in the most generality. Hence we have to consider the additional suitable conditions to establish a Bernstein type result for higher codimension.

In recent years, remarkable progress has been made by [5], [6], [8], [10] and [11] in Bernstein type problems of minimal submanifolds with higher codimension and special Lagrangian submanifolds. The key idea in these papers is to find a suitable subharmonic function, whose vanishing implies the minimal graph is totally geodesic. Let $M$ be a minimal submanifold of $R^{n+m}$ that can be represented as the graph of a smooth map $f: R^{n} \rightarrow R^{m}$. The function is given by

$$
* \Omega=\frac{1}{\sqrt{\operatorname{det}\left(I+(d f)^{t} d f\right)}}
$$

Received August 17, 2007.

Revised May 16, 2008.

Accepted June 27, 2008.

2000 Mathematics Subject Classification: Primary 53A10, 53A07, 53C38.

Supported by the Zhongdian grant of NSFC. 
Jost-Xin [5] established a Bernstein result for $M$ under the condition $* \Omega \geq$ $K>1 / 2$, which improves the previous results in [3] and [2]. Wang in [10] derived a nice Bochner type formula for the function $\ln (* \Omega)^{-1}$. Under the so-called area-decreasing condition, he obtained a Bernstein result for higher codimension case too.

Due to string theory, special Lagrangian submanifolds received much attention in recent years. Some authors also tried to establish Bernstein type results for special Lagrangian submanifolds (see [6], [8] and [11]). It is known that a special Lagrangian graph may be represented by a gradient of a smooth function, i.e., $f=\nabla u$ for a smooth function $u: R^{n} \rightarrow R$. The function $u$ is called the potential function. Tsui and Wang in [8] obtained Bernstein results for special Lagrangian graphs by applying the same Bochner formula. We should point out that the same formula for special Lagrangian graphs was also derived by Yuan in [11] from a different point of view. An important technique used by Yuan is the so-called Lewy transformation which allows him to prove: Any special Lagrangian graph given by a convex potential function must be an affine plane. Actually, Yuan obtained a little bit stronger Bernstein result for special Lagrangian graph under the condition $\operatorname{Hess}(u) \geq-\epsilon(n) I$, where $\epsilon(n)$ is a small dimensional constant.

In this paper, we will investigate a real minimal Lagrangian graph $\Sigma^{n}$ in Quaternion space $H^{n} \cong R^{4 n}$ which is given by three potential functions $u_{s}: R^{n} \rightarrow R, s=1,2,3$ as follows:

$$
\Sigma=\left\{\left(x, \nabla u_{1}, \nabla u_{2}, \nabla u_{3}\right): x \in R^{n}\right\} .
$$

The Lagrangian condition forces the three matrices Hess $\left(u_{s}\right)$ to be commutative with each other. As a result, we may choose a particular quaternionic frame corresponding to singular value decomposition of $\nabla u_{s}(s=1,2,3)$ at each point. A useful formula for the minimal Lagrangian graph is derived by applying Wang's Bochner formula to the quaternionic frame. Using this formula, we obtain some Bernstein theorems for $\Sigma$, which generalize those results in [8] and [11] (see Section 3 for details). Obviously, when $u_{2}$ and $u_{3}$ are constant, $\Sigma$ is just the special Lagrangian graph in $C^{n}$. By combining Wang's result in [8] and a Lewy transformation, we get a new Bernstein Theorem for special Lagrangian graph too. Note that our lower bound for $\operatorname{Hess}(u)$ is independent of the dimension. Finally, we consider the minimal Lagrangian graph given by three same potential functions, i.e., $\nabla u_{s}=\nabla u$ $(s=1,2,3)$. In this case, we also find a suitable lewy transformation to prove a Bernstein result similar to the above mentioned results. 


\section{§2. Preliminaries}

We first recall a formula derived in [9], [10]. Let $\Sigma$ be an oriented $n$ dimensional submanifold of $R^{n+m}$ and $\Omega$ a parallel $n$ form on $R^{n+m}$. Around any point $p \in \Sigma$, we choose any oriented orthonormal frames $\left\{e_{i}\right\}_{i=1}^{n}$ for $T_{p} \Sigma$ and $\left\{e_{\alpha}\right\}_{\alpha=n+1}^{n+m}$ for $N_{p} \Sigma$, the normal bundle of $\Sigma$. The second fundamental form of $\Sigma$ is denoted by $h_{\alpha i j}=\left\langle\nabla_{e_{i}} e_{j}, e_{\alpha}\right\rangle$. If we assume $\Sigma$ has parallel mean curvature vector, then the global function $* \Omega=\Omega\left(e_{1}, \ldots, e_{n}\right)$ satisfies (see [9], [10]):

$$
\begin{gathered}
\triangle * \Omega+* \Omega\left(\sum_{\alpha l k} h_{\alpha, l, k}^{2}\right)-2 \sum_{\alpha, \beta, k}\left[\Omega_{\alpha \beta 3 \cdots n} h_{\alpha 1 k} h_{\beta 2 k}+\cdots\right. \\
\left.\cdots+\Omega_{1 \cdots(n-2) \alpha \beta} h_{\alpha(n-1) k} h_{\beta n k}\right]=0 \\
(* \Omega)_{k}=\sum_{\alpha} \Omega_{\alpha 2 \cdots n} h_{\alpha 1 k}+\cdots+\Omega_{1 \cdots(n-1) \alpha} h_{\alpha n k} \\
1 \leq i, j, k, \ldots \leq n, \quad n+1 \leq \alpha, \beta \leq n+m
\end{gathered}
$$

where $\triangle$ is the Laplace operator of the induced metric on $\Sigma$ and $\Omega_{\alpha \beta 3 \cdots n}=$ $\Omega\left(e_{\alpha}, e_{\beta}, e_{3}, \ldots, e_{n}\right)$, etc.

Let $g$ be the standard Euclidean metric on $H^{n} \cong R^{4 n}$ which is Kähler with respect to the three natural complex structures $I, J, K$ on $R^{4 n}$. Set $\omega_{I}=g(I),, \omega_{J}=g(J$,$) and \omega_{K}=g(K$,$) . An n$-dimensional submanifold $f: \Sigma^{n} \rightarrow R^{4 n}$ is called Lagrangian if it satisfies:

$$
f^{*} \omega_{I}=f^{*} \omega_{J}=f^{*} \omega_{K}=0 .
$$

Suppose $\Sigma$ is a graph defined by $f=\left(f_{1}, f_{2}, f_{3}\right)$, where $f_{s}=\left(f_{s}^{1}, \ldots, f_{s}^{n}\right)$ : $R^{n} \rightarrow R^{n} s=1,2,3$ are smooth maps. It is easy to see that $\Sigma$ is Lagrangian if and only if $f_{1}, f_{2}, f_{3}$ satisfy:

$$
\left\{\begin{array}{l}
-\frac{\partial f_{1}^{j}}{\partial x_{i}}+\frac{\partial f_{1}^{i}}{\partial x_{j}}+\sum_{k=1}^{n}\left\{\frac{\partial f_{2}^{k}}{\partial x_{i}} \frac{\partial f_{3}^{k}}{\partial x_{j}}-\frac{\partial f_{2}^{k}}{\partial x_{j}} \frac{\partial f_{3}^{k}}{\partial x_{i}}\right\}=0 \\
-\frac{\partial f_{2}^{j}}{\partial x_{i}}+\frac{\partial f_{2}^{i}}{\partial x_{j}}+\sum_{k=1}^{n}\left\{\frac{\partial f_{3}^{k}}{\partial x_{i}} \frac{\partial f_{1}^{k}}{\partial x_{j}}-\frac{\partial f_{3}^{k}}{\partial x_{j}} \frac{\partial f_{1}^{k}}{\partial x_{i}}\right\}=0 \\
-\frac{\partial f_{3}^{j}}{\partial x_{i}}+\frac{\partial f_{3}^{i}}{\partial x_{j}}+\sum_{k=1}^{n}\left\{\frac{\partial f_{1}^{k}}{\partial x_{i}} \frac{\partial f_{2}^{k}}{\partial x_{j}}-\frac{\partial f_{1}^{k}}{\partial x_{j}} \frac{\partial f_{2}^{k}}{\partial x_{i}}\right\}=0
\end{array}\right.
$$

where $i, j \in\{1, \ldots, n\}$. Obviously, if $f_{s}=\nabla u_{s}$ for some smooth functions $u_{s}: R^{n} \rightarrow R(s=1,2,3)$, then $\Sigma$ is Lagrangian if and only if $u_{s}, s=1,2,3$ 
satisfy:

$$
\operatorname{Hess}\left(u_{s}\right) \operatorname{Hess}\left(u_{t}\right)=\operatorname{Hess}\left(u_{t}\right) \operatorname{Hess}\left(u_{s}\right) \text { for all } s, t \in\{1,2,3\} \text {. }
$$

From now on, we will consider minimal Lagrangian graph given by the three potential functions $u_{s}, s=1,2,3$. Note that if $u_{2}, u_{3}$ are constants, $\Sigma$ is just the special Lagrangian graph considered in [6], [8] and [11].

EXAMPLE 1. The smallest interesting dimension is $n=2$. We would like to give some examples of minimal Lagrangian surfaces in $H^{2}=R^{8}$.

First, let $u_{s}\left(x_{1}, x_{2}\right), s=1,2,3$ be harmonic functions on $R^{2}$. We see that $\left(u_{s}\right)_{x_{1}}-\sqrt{-1}\left(u_{s}\right)_{x_{2}}$ is a holomorphic function of $z=x_{1}+\sqrt{-1} x_{2}$ for each $s$. It follows that the graph $\Sigma=\left\{\left(x_{1}, x_{2}, \nabla u_{1}, \nabla u_{2}, \nabla u_{3}\right): x=\right.$ $\left.\left(x_{1}, x_{2}\right) \in R^{2}\right\}$ is a holomorphic curve in $C^{4}=R^{8}$, and thus a minimal surfaces in $R^{8}$. In particular, if $u$ is a harmonic function on $R^{2}$, we have minimal Lagrangian graphs $\Sigma_{1}=\left\{(x, \nabla u, x, \nabla u): x \in R^{2}\right\}$ and $\Sigma_{2}=$ $\left\{(x, \nabla u, \nabla u, \nabla u): x \in R^{2}\right\}$.

From Example 1, we know that there exist many minimal graphic Lagrangian submanifolds of $R^{4 n}$.

\section{§3. Main results for minimal Lagrangian graphs}

Let $\Sigma=\left(x, \nabla u_{1}, \nabla u_{2}, \nabla u_{3}\right)$ be an $n$ dimensional minimal Lagrangian submanifold in $R^{4 n}$. From the previous section, we know that $\left\{u_{s}\right\}_{s=1,2,3}$ satisfy (4) at each point $x \in R^{n}$. So we may diagonalize $\operatorname{Hess}\left(u_{s}\right), s=1,2,3$ simultaneously at each point $x$ via the singular decomposition, that is, there exist orthonormal bases $\left\{a_{i}\right\}_{i=1, \ldots, n}$ for $R^{n}$ and $\left\{a_{\alpha}\right\}_{\alpha=n+1, \ldots, 4 n}$ for $R^{3 n}$ such that

$$
\operatorname{Hess}\left(u_{s}\right) a_{i}=\lambda_{i}^{(s)} a_{s n+i}
$$

and

$$
I a_{i}=a_{n+i}, \quad J a_{i}=a_{2 n+i}, \quad K a_{i}=a_{3 n+i}
$$

for $i=1, \ldots, n$. Set

$$
\begin{aligned}
e_{i} & =\frac{a_{i}+\lambda_{i}^{(1)} a_{n+i}+\lambda_{i}^{(2)} a_{2 n+i}+\lambda_{i}^{(3)} a_{3 n+i}}{A_{i}} \\
e_{n+i} & =\frac{-\lambda_{i}^{(1)} a_{i}+a_{n+i}-\lambda_{i}^{(3)} a_{2 n+i}+\lambda_{i}^{(2)} a_{3 n+i}}{A_{i}}
\end{aligned}
$$




$$
\begin{aligned}
e_{2 n+i} & =\frac{-\lambda_{i}^{(2)} a_{i}+\lambda_{i}^{(3)} a_{n+i}+a_{2 n+i}-\lambda_{i}^{(1)} a_{3 n+i}}{A_{i}} \\
e_{3 n+i} & =\frac{-\lambda_{i}^{(3)} a_{i}-\lambda_{i}^{(2)} a_{n+i}+\lambda_{i}^{(1)} a_{2 n+i}+a_{3 n+i}}{A_{i}}
\end{aligned}
$$

where $A_{i}=\sqrt{1+\left(\lambda_{i}^{(1)}\right)^{2}+\left(\lambda_{i}^{(2)}\right)^{2}+\left(\lambda_{i}^{(3)}\right)^{2}}$. Note that, $e_{n+i}=I e_{i}, e_{2 n+i}=$ $J e_{i}$ and $e_{3 n+i}=K e_{i}$ at the corresponding point $p=\left(x, \nabla u_{1}(x), \nabla u_{2}(x)\right.$, $\left.\nabla u_{3}(x)\right)$. Thus we have an orthonormal frame $\left\{e_{i}\right\}_{i=1, \ldots, n}$ for $T_{p} \Sigma$ and $\left\{e_{n+i}, e_{2 n+i}, e_{3 n+i}\right\}_{i=1, \ldots, n}$ for $N_{p} \Sigma$. Define the second fundamental form of $\Sigma$ as follows:

$$
h_{i j k}^{(s)}=\left\langle\widetilde{\nabla}_{e_{i}} e_{j}, e_{n s+k}\right\rangle, \quad s=1,2,3 .
$$

Since $\widetilde{\nabla} I=\widetilde{\nabla} J=\widetilde{\nabla} K=0$ and $\Sigma$ is Lagrangian, we know that $h_{i j k}^{(s)}$ is symmetric in $i, j, k$. Now take $\Omega=d x_{1} \wedge \cdots \wedge d x_{n}$. It is not hard to see

$$
* \Omega=\frac{1}{\sqrt{\prod_{i=1}^{n}\left(1+\sum_{s=1}^{3}\left(\lambda_{i}^{(s)}\right)^{2}\right)}} .
$$

By applying the formula (1) to the above quaternionic frame $\left\{e_{i}, e_{n+i}, e_{2 n+i}\right.$, $\left.e_{3 n+i}\right\}_{i=1, \ldots, n}$, we get

Proposition 3.1. Let $\Sigma=\left(x, \nabla u_{1}(x), \nabla u_{2}(x), \nabla u_{3}(x)\right)$ be a minimal graph in $R^{4 n}$ and $\left\{\lambda_{i}^{(s)}\right\}$ be the eigenvalues of $\operatorname{Hess}\left(u_{s}\right), s=1,2,3$. Then $* \Omega$ satisfies

$$
\begin{aligned}
\triangle * \Omega=-* \Omega\left\{\sum_{s=1}^{3} \sum_{i j k=1}^{n}\left(h_{i j k}^{(s)}\right)^{2}-2 \sum_{s t=1}^{3} \sum_{k, i<j} \lambda_{i}^{(s)} \lambda_{j}^{(t)} h_{i i k}^{(s)} h_{j j k}^{(t)}\right. & \\
& \left.+2 \sum_{s t=1}^{3} \sum_{k, i<j} \lambda_{i}^{s} \lambda_{j}^{(t)} h_{i j k}^{(s)} h_{i j k}^{(t)}\right\}
\end{aligned}
$$

where $\triangle$ is the Laplace operator of the induced metric on $\Sigma$.

Now we shall calculate

$$
\triangle(\ln * \Omega)=\frac{* \Omega \triangle(* \Omega)-|\nabla * \Omega|^{2}}{|* \Omega|^{2}} .
$$

By formula (2), the covariant derivative of $* \Omega$ is

$$
(* \Omega)_{k}=-* \Omega\left(\sum_{s=1}^{3} \sum_{i=1}^{n} \lambda_{i}^{(s)} h_{i i k}^{s}\right) .
$$


Plug this and equation (5) into equation (6) and we obtain:

$$
\triangle\left[\ln (* \Omega)^{-1}\right]=\sum_{s=1}^{3} \sum_{i j k=1}^{n}\left(h_{i j k}^{(s)}\right)^{2}+\sum_{s t=1}^{3} \sum_{i j k=1}^{n} \lambda_{i}^{(s)} \lambda_{j}^{(t)} h_{i j k}^{(s)} h_{i j k}^{(t)} .
$$

Set $\Lambda_{i}=\left(\lambda_{i}^{(1)}, \lambda_{i}^{(2)}, \lambda_{i}^{(3)}\right)$ and $h_{i j k}=\left(h_{i j k}^{(1)}, h_{i j k}^{(2)}, h_{i j k}^{(3)}\right)$. So

$$
\triangle \ln [* \Omega]^{(-1)}=\left\{\sum_{i j k=1}^{n} h_{i j k}\left(I+\Lambda_{i}^{T} \Lambda_{j}\right) h_{i j k}^{T}\right\} .
$$

We may rewrite (9) as

$$
\triangle \ln [* \Omega]^{(-1)}=\frac{1}{3}\left\{\sum_{i j k=1}^{n} h_{i j k}\left(3 I+\Lambda_{i}^{T} \Lambda_{j}+\Lambda_{j}^{T} \Lambda_{k}+\Lambda_{k}^{T} \Lambda_{i}\right) h_{i j k}^{T}\right\} .
$$

Set $S_{i j}=\frac{1}{2}\left(\Lambda_{i}^{T} \Lambda_{j}+\Lambda_{j}^{T} \Lambda_{i}\right)$. We have the following:

ThEOREM 3.2. Let $\Sigma=\left(x, \nabla u_{1}, \nabla u_{2}, \nabla u_{3}\right)$ be an $n$-dimensional minimal Lagrangian submanifold of $R^{4 n}$. If there exist $\delta, K>0$ such that

$$
\left|\lambda_{i}^{(s)}\right| \leq K, \quad \text { and } \quad S_{i j}+S_{j k}+S_{k i} \geq(-3+\delta) I
$$

for $i, j, k \in\{1, \ldots, n\}, s \in\{1,2,3\}$, then $\Sigma$ is an affine plane.

Proof. Set

$$
\begin{aligned}
F_{i j k}(X) & =X\left(3 I+\Lambda_{i}^{T} \Lambda_{j}+\Lambda_{j}^{T} \Lambda_{k}+\Lambda_{k}^{T} \Lambda_{i}\right) X^{T} \\
& =X\left(3 I+S_{i j}+S_{j k}+S_{k i}\right) X^{T}
\end{aligned}
$$

for fixed $i, j, k$ and any $X \in R^{3}$. By the assumption we have

$$
F_{i j k}(X) \geq \delta\|X\|^{2} \text {. }
$$

From (10) we have

$$
\triangle \ln [* \Omega]^{(-1)}=\frac{1}{3} \sum_{i j k=1}^{n} F_{i j k}\left(h_{i j k}\right) \geq \frac{1}{3} \sum_{i j k=1}^{n} \delta\left\|h_{i j k}\right\|^{2}=\frac{1}{3} \delta\|A\|^{2}
$$

where $A$ is the second fundamental form of $\Sigma$. Note that $\left|\lambda_{i}^{(s)}\right| \leq K$ means $\Sigma$ is of bounded slope. So we may perform blow down to get a minimal Lagrangian cone. Obviously the minimal Lagrangian cone also satisfies the assumption. By applying maximum principle we conclude that the minimal cone is flat and then Allard regularity theorem implies that $\Sigma$ is an affine plane. 
Corollary 3.3. Let $\Sigma=\left(x, \nabla u_{1}, \nabla u_{2}, \nabla u_{3}\right)$ be a minimal Lagrangian submanifold of $R^{4 n}$. If there exist $\delta, K>0$ such that

$$
\left|\lambda_{i}^{(s)}\right| \leq K, \quad \text { and } \quad S_{i j} \geq\left(-\frac{3}{2}+\delta\right) I
$$

where $i, j \in\{1, \ldots, n\}, s \in\{1,2,3\}$, then $\Sigma$ is an affine plane.

Proof.

$$
\begin{aligned}
F_{i j k}(X) & =X\left(3 I+\Lambda_{i}^{T} \Lambda_{j}+\Lambda_{j}^{T} \Lambda_{k}+\Lambda_{k}^{T} \Lambda_{i}\right) X^{T} \\
& =3\|X\|^{2}+X S_{i j} X^{T}+X S_{j k} X^{T}+X S_{j k} X^{T} .
\end{aligned}
$$

It is easy to know that

$$
\begin{aligned}
X S_{i j} X^{T} & =\left(X, \Lambda_{i}\right)\left(\Lambda_{j}, X\right), \\
X S_{j k} X^{T} & =\left(X, \Lambda_{j}\right)\left(\Lambda_{k}, X\right), \\
X S_{k i} X^{T} & =\left(X, \Lambda_{k}\right)\left(\Lambda_{i}, X\right) .
\end{aligned}
$$

Observe that one of $\left(X, \Lambda_{i}\right)\left(X, \Lambda_{j}\right),\left(X, \Lambda_{j}\right)\left(X, \Lambda_{k}\right),\left(X, \Lambda_{k}\right)\left(X, \Lambda_{i}\right)$ must be nonnegative. From the assumption, we know

$$
F_{i j k}(X) \geq 2 \delta\|X\|^{2}
$$

i.e.

$$
S_{i j}+S_{j k}+S_{k i} \geq(-3+2 \delta) I .
$$

The conclusion follows immediately from Theorem 3.2.

Remark 3.4. When $u_{1}$ and $u_{2}$ are constants, we can recover Wang's result in [8] for special Lagrangian submanifolds.

COROllary 3.5. Let $\Sigma=\left(x, \nabla u_{1}, \nabla u_{2}, \nabla u_{3}\right)$ be a minimal Lagrangian submnanifold of $R^{4 n}$. If there exists a small positive number $\delta$ such that

$$
\left|\Lambda_{i}\right| \leq \sqrt{\frac{3}{2}-\delta}
$$

for $i \in\{1, \ldots, n\}$, then $\Sigma$ is an affine plane. 
Proof. By the assumption $\left|\Lambda_{i}\right| \leq \sqrt{\frac{3}{2}-\delta}$ for $i=1, \ldots, n$ and the Cauchy-Schwarz inequality, we have

$$
X S_{i j} X^{T}=\left(X, \Lambda_{i}\right)\left(X, \Lambda_{j}\right) \geq\left(-\frac{3}{2}+\delta\right)\|X\|^{2}
$$

for any fixed $i, j \in\{1, \ldots, n\}$ and $X \in R^{3}$. So the symmetric matrix $S_{i j}$ satisfies

$$
S_{i j} \geq\left(-\frac{3}{2}+\delta\right) I
$$

Therefore the conclusion follows immediately from Corollary 3.3.

Remark 3.6. If $u_{2}$ and $u_{3}$ are constants, then $\Sigma=\left(x, \nabla u_{1}(x), 0,0\right)$, which may be regarded as a minimal Lagrangian graph $\Sigma=\left(x, \nabla u_{1}(x)\right)$ in $C^{n}$. So the above Corollary generalizes those results in [8] and [11].

In the following, we will consider two special kinds of minimal Lagrangian graphs: $\Sigma=(x, \nabla u, 0,0)$ or $\Sigma=(x, \nabla u, \nabla u, \nabla u)$ in $H^{n}$. We have already pointed out that the previous case is just the special Lagrangian graph.

THEOREM 3.7. Let $\Sigma=(x, \nabla u)$ be a minimal Lagrangian submanifold of $C^{n}$. If there exists a positive constant $C<\sqrt{6} / 12$ such that

$$
\operatorname{Hess}(u) \geq-C I
$$

then $\Sigma$ is an affine plane.

Proof. We identify $C$ with $R^{2}$ as follows:

$$
C \ni x+\sqrt{-1} y \longleftrightarrow(x, y) \in R^{2} .
$$

For $a+\sqrt{-1} b \in S U(1)$, its real representation matrix on $R^{2}$ is given by

$$
A=\left(\begin{array}{cc}
a & -b \\
b & a
\end{array}\right)
$$

where $a^{2}+b^{2}=1$.

We consider the transformation $A^{(n)}=(A, \ldots, A)$ on $\underbrace{C \times \cdots \times C}_{n}=$ $R^{2 n}$ defined by

$$
\left\{\begin{array}{l}
\bar{x}=a x+b y \\
\bar{y}=-b x+a y
\end{array}\right.
$$


where $(x, y),(\bar{x}, \bar{y}) \in R^{n} \times R^{n}=C^{n}$. Set $a=h / \sqrt{1+h^{2}}$ and $b=1 / \sqrt{1+h^{2}}$, where $h$ is a constant to be chosen. It follows that $\Sigma$ has a new parametrization

$$
\left\{\begin{array}{l}
\bar{x}=\frac{1}{\sqrt{1+h^{2}}}(h x+\nabla u) \\
\bar{y}=\frac{1}{\sqrt{1+h^{2}}}(-x+h \nabla u)
\end{array}\right.
$$

Since $u+\frac{1}{2} C\|x\|^{2}$ is a convex function, we have

$$
\begin{aligned}
& \left|\bar{x}_{1}-\bar{x}_{2}\right|^{2}=\frac{1}{1+h^{2}}\left|h x_{2}+\nabla u\left(x_{2}\right)-h x_{1}-\nabla u\left(x_{1}\right)\right|^{2} \\
& =\frac{1}{1+h^{2}}\left|(h-C)\left(x_{2}-x_{1}\right)+\left(\nabla u\left(x_{2}\right)+C x_{2}\right)-\left(\nabla u\left(x_{1}\right)+C x_{1}\right)\right|^{2} \\
& =\frac{1}{1+h^{2}}\left\{(h-C)^{2}\left|x_{2}-x_{1}\right|^{2}\right. \\
& \quad+2(h-C)\left(x_{2}-x_{1}\right)\left[\left(\nabla u\left(x_{2}\right)+C x_{2}\right)-\left(\nabla u\left(x_{1}\right)+C x_{1}\right)\right] \\
& \left.\quad+\left|\left(\nabla u\left(x_{2}\right)+C x_{2}\right)-\left(\nabla u\left(x_{1}\right)+C x_{1}\right)\right|^{2}\right\} \\
& \geq \frac{1}{1+h^{2}}(h-C)^{2}\left|x_{2}-x_{1}\right|^{2} .
\end{aligned}
$$

Now we assume $h>C$. Then (13) implies that $\Sigma$ is still a graph over the whole $\bar{x}$ space $R^{n}$. Further $\Sigma$ is still a Lagrangian graph over $\bar{x}$, that means $\Sigma$ has the representation $(\bar{x}, \nabla \bar{u}(\bar{x}))$ with a potential function $\bar{u} \in C^{\infty}\left(R^{n}\right)$. We may derive from (12) that

$$
\operatorname{Hess}(\bar{u}(\bar{x}))=(h I+\operatorname{Hess}(u(x)))^{-1}(-I+h \operatorname{Hess}(u(x))) .
$$

From $\operatorname{Hess}(u) \geq-C I$, we see that

$$
-\frac{1+h C}{h-C} I \leq \operatorname{Hess}(\bar{u}) \leq h I .
$$

By solving $h=\frac{1+h C}{h-C}$, we get $h=C+\sqrt{C^{2}+1}$ which obviously satisfies the previous assumption $h>C$. So (14) becomes

$$
-\left(C+\sqrt{C^{2}+1}\right) I \leq \operatorname{Hess}(\bar{u}(\bar{x})) \leq\left(C+\sqrt{C^{2}+1}\right) I .
$$

The condition $C \leq \sqrt{6} / 12$ implies that any eigenvalue $\lambda$ of $\operatorname{Hess}(\bar{u})$ satisfies

$$
|\lambda| \leq C+\sqrt{C^{2}+1}<\sqrt{\frac{3}{2}} .
$$

From Corollary 3.5, we know that $\Sigma$ is an affine plane. 
Remark 3.8. When $n=3$, Yuan [11] showed that the lower bound for $\operatorname{Hess}(u)$ in Bernstein Theorem can be relaxed to be an arbitrary constant.

THEOREM 3.9. Let $\Sigma=(x, \nabla u, \nabla u, \nabla u)$ be a minimal Lagrangian submanifold of $R^{4 n}$. If there exists a positive constant $C<\sqrt{2} / 12$ such that

$$
\operatorname{Hess}(u) \geq-C I
$$

then $\Sigma$ is an affine plane.

Proof. We identify $H$ with $C^{2}$ as follows:

$H \ni x+I y+J z+K w=(x+J z)+I(y+J w) \longleftrightarrow(x+J z, y+J w) \in C^{2}$.

For a matrix $M=A+j B \in S U(2)$, its real representation on $R^{4}$ is given by

$$
M\left(\begin{array}{c}
x \\
y \\
z \\
w
\end{array}\right)=\left(\begin{array}{cc}
A & -B \\
B & A
\end{array}\right)\left(\begin{array}{c}
x \\
y \\
z \\
w
\end{array}\right)
$$

with $A A^{T}+B B^{T}=I_{2}$ and $A B^{T}=B A^{T}$. Obviously if $a^{2}+3 b^{2}=1$, then

$$
D=\left(\begin{array}{cccc}
a & b & b & b \\
-b & a & b & -b \\
-b & -b & a & b \\
-b & b & -b & a
\end{array}\right) \in S U(2)=S p(1)
$$

We consider the transformation $D^{(n)}=(D, \ldots, D)$ on $\underbrace{H \times \cdots \times H}_{n}=H^{n}=$ $R^{4 n}$ defined by

$$
\left\{\begin{aligned}
\bar{x} & =a x+b y+b z+b w \\
\bar{y} & =-b x+a y+b z-b w \\
\bar{z} & =-b x-b y+a z+b w \\
\bar{w} & =-b x+b y-b z+a w
\end{aligned}\right.
$$

where $(x, y, z, w),(\bar{x}, \bar{y}, \bar{z}, \bar{w}) \in R^{n} \times R^{n} \times R^{n} \times R^{n}=R^{4 n}$. Set $a=h / \sqrt{1+h^{2}}$ and $\sqrt{3} b=1 / \sqrt{1+h^{2}}$, where $h$ is a constant to be chosen. It follows that 
$\Sigma$ has a new parametrization

$$
\left\{\begin{array}{l}
\bar{x}=\frac{1}{\sqrt{1+h^{2}}}(h x+\sqrt{3} \nabla u) \\
\bar{y}=\frac{1}{\sqrt{1+h^{2}}}\left(-\frac{1}{\sqrt{3}} x+h \nabla u\right) \\
\bar{z}=\frac{1}{\sqrt{1+h^{2}}}\left(-\frac{1}{\sqrt{3}} x+h \nabla u\right) \\
\bar{w}=\frac{1}{\sqrt{1+h^{2}}}\left(-\frac{1}{\sqrt{3}} x+h \nabla u\right)
\end{array}\right.
$$

Since $u+\frac{1}{2} C\|x\|^{2}$ is a convex function, we have

$$
\begin{aligned}
& \left|\bar{x}_{1}-\bar{x}_{2}\right|^{2}=\frac{1}{1+h^{2}}\left|h x_{2}+\sqrt{3} \nabla u\left(x_{2}\right)-h x_{1}-\sqrt{3} \nabla u\left(x_{1}\right)\right|^{2} \\
& =\frac{1}{1+h^{2}}\left|(h-\sqrt{3} C)\left(x_{2}-x_{1}\right)+\sqrt{3}\left[\left(\nabla u\left(x_{2}\right)+C x_{2}\right)-\left(\nabla u\left(x_{1}\right)+C x_{1}\right)\right]\right|^{2} \\
& =\frac{1}{1+h^{2}}\left\{\left[(h-\sqrt{3} C)^{2}\left|x_{2}-x_{1}\right|^{2}\right]\right. \\
& \quad+2 \sqrt{3}(h-\sqrt{3} C)\left(x_{2}-x_{1}\right)\left[\left(\nabla u\left(x_{2}\right)+C x_{2}\right)-\left(\nabla u\left(x_{1}\right)+C x_{1}\right)\right] \\
& \left.\quad+3\left|\left(\nabla u\left(x_{2}\right)+C x_{2}\right)-\left(\nabla u\left(x_{1}\right)+C x_{1}\right)\right|^{2}\right\} \\
& \geq \frac{1}{1+h^{2}}(h-\sqrt{3} C)^{2}\left|x_{2}-x_{1}\right|^{2} .
\end{aligned}
$$

Now we assume $h>\sqrt{3} C$. Then (18) implies that $\Sigma$ is still a graph over the whole $\bar{x}-R^{n}$, that is $\Sigma$ has the representation $\left(\bar{x}, f_{1}(\bar{x}), f_{2}(\bar{x}), f_{3}(\bar{x})\right)$. Since $\Sigma$ is still minimal Lagrangian, we see from (3) that $f=\nabla \bar{u}$, that is, $\Sigma=(\bar{x}, \nabla \bar{u}, \nabla \bar{u}, \nabla \bar{u})$ for some function $\bar{u} \in C^{\infty}\left(R^{n}\right)$. We may derive from (17) that

$$
\operatorname{Hess}(\bar{u}(\bar{x}))=(h I+\sqrt{3} \operatorname{Hess}(u(x)))^{-1}\left(-\frac{1}{\sqrt{3}} I+h \operatorname{Hess}(u(x))\right) .
$$

From $\operatorname{Hess}(u) \geq-C I$, we see that

$$
-\frac{\frac{1}{\sqrt{3}}+h C}{h-\sqrt{3} C} I \leq \operatorname{Hess}(\bar{u}) \leq \frac{h}{\sqrt{3}} I .
$$


By solving $\frac{h}{\sqrt{3}}=\frac{\frac{1}{\sqrt{3}}+h C}{h-\sqrt{3} C}$, we get $h=\sqrt{3} C+\sqrt{3 C^{2}+1}$ which obviously satisfies the previous assumption $h>\sqrt{3} C$. So (19) becomes

$$
-\frac{\sqrt{3} C+\sqrt{3 C^{2}+1}}{\sqrt{3}} I \leq \operatorname{Hess}(\bar{u}) \leq \frac{\sqrt{3} C+\sqrt{3 C^{2}+1}}{\sqrt{3}} I .
$$

The condition $C<\sqrt{2} / 12$ implies that any eigenvalue $\lambda$ of $\operatorname{Hess}(\bar{u}(\bar{x}))$ satisfies

$$
|\lambda| \leq \frac{\sqrt{3} C+\sqrt{3 C^{2}+1}}{\sqrt{3}}<\sqrt{\frac{1}{2}} .
$$

Then the singular values $\Lambda_{i}=\left(\lambda_{i}, \lambda_{i}, \lambda_{i}\right)$ of $\Sigma=(\bar{x}, \nabla \bar{u}(\bar{x}), \nabla \bar{u}(\bar{x}), \nabla \bar{u}(\bar{x}))$ satisfy:

$$
\left|\Lambda_{i}\right| \leq \sqrt{3} C+\sqrt{3 C^{2}+1}<\sqrt{\frac{3}{2}} \text { for } i \in\{1, \ldots, n\} .
$$

Hence, by Corollary 3.5, we know that $\Sigma$ is an affine plane.

Corollary 3.10. Let $\Sigma=(x, \nabla u, \nabla u, \nabla u)$ be a minimal Lagrangian submanifold of $R^{4 n}$. If $u$ is a smooth convex function on $R^{n}$, then $\Sigma$ is an affine plane.

Acknowledgement. We would like to thank Professors Gu, C.H. and $\mathrm{Hu}$, H.S. for their valuable suggestions and constant encouragement. We thank Prof. Y.L. Xin for his helpful comments. We would also like to thank the referee for his valuable suggestions.

\section{REFERENCES}

[1] K. Ecker and G. Huisken, A Bernstein result for minimal graphs of controlled growth, J. Differential Geom., 31 (1990), no. 2, 397-400.

[2] S. Hildebrant, J. Jost and K.-O. Widman, Harmonic mappings and minimal submanifolds, Invent. Math., 62 (1980/81), no. 2, 269-298.

[3] D. Fischer-Colbrie, Some rigidity theorems for minimal submanifolds of the sphere, Acta Math., 145 (1980), no. 1, 29-46.

[4] R. Harvey and H. B. Lawson, Calibrated geometries, Acta Math., 148 (1982), 47-157.

[5] J. Jost and Y. L. Xin, Bernstein type theorems for higher codimension, Calc. Var. Partial Differential Equations, 9 (1999), no. 4, 277-296.

[6] J. Jost and Y. L. Xin, A Bernstein Theorem for Special Lagrangian Graphs, Calc. Var. and Partial Differential Equations, 15 (2002), 299-312.

[7] B. Lawson and R. Osserman, Non-exsitence, non-uniqueness and irregularity of solutions to minimal surface system, Acta Math., 139 (1970), 1-17. 
[8] M.-P. Tsui and M.-T. Wang, A Berstein Type Result for Special Lagrangian Submanifolds, Math. Res. Lett., 9 (2002), no. 4, 529-535.

[9] M.-T. Wang, Long-time exsitence and convergence of graghic mean curvature in arbitrary codimension, Invent. Math., 148 (2002), no. 3, 525-543.

[10] M.-T. Wang, On graphic Berstein type results in higher codimensions, Trans. Amer. Math. Soc., 355 (2003), no. 1, 265-271.

[11] Y. Yuan, A Bernstein problem for special Lagrangian equaton, Invent. Math., 150 (2002) no. 1, 117-125.

Yuxin Dong

Institute of Mathematics

Fudan University

Shanghai, 200433

P. R. China

And Key Laboratory of Mathematics for Nonlinear Sciences (Fudan University) Ministry of Education

yxdong@fudan.edu.cn

Yingbo Han

Department of Mathematics

Southeast University

Nanjing, 211189

P. R. China

yingbohan@163.com

Qingchun Ji

Institute of Mathematics

Fudan University

Shanghai, 200433

P. R. China

And Key Laboratory of Mathematics for Nonlinear Sciences (Fudan University) Ministry of Education

qingchunji@fudan.edu.cn 